\title{
ATUAÇÃO DO ENFERMEIRO NO CONTROLE DE FATORES DE RISCOS E PREVENÇÃO DA INFECÇÃO DE SÍTIO CIRÚRGICO: UMA REVISÃO INTEGRATIVA
}

\author{
Adilma da Cunha Cavalcanti ${ }^{1}$, Jéssyka Jordana Guimarães Freitas ${ }^{1}$, Julyana \\ Falcão Madeira ${ }^{1}$, José Tarcisio de Azevedo Sales ${ }^{1}$, Mariana Albernaz Pinheiro \\ de Carvalho? \\ ${ }^{1}$ Curso de Bacharelado em Enfermagem, Unidade Acadêmica de Saúde, Universidade Federal \\ de Campina Grande, Cuité-PB, Brasil. \\ ${ }^{2}$ Prof ${ }^{a}$ Unidade Acadêmica de Enfermagem, Universidade Federal de Campina Grande, Cuité, \\ PB, Brasil. \\ Email para correspondência: adilmacavalcanti@yahoo.com.br
}

\begin{abstract}
Resumo
As Infecções de Sítio Cirúrgico (ISC) são agravos que acometem pacientes submetidos à algum procedimento operatório e representam $15 \%$ das infecções hospitalares. Objetivou-se caracterizar a produção científica nacional e internacional sobre o tema, discutindo intervenções e cuidados de enfermagem na perspectiva da prevenção da infecção do sítio cirúrgico (ISC).Trata-se de uma revisão integrativa construída a partir de um levantamento bibliográfico de 2275 materiais nas bases de dados: BDENF; IBECS; LILACS; MEDLINE e SCIELO, a partir dos critérios de inclusão: artigos com texto completos, disponíveis e online na língua vernácula, inglesa ou espanhola, publicados entre 2014 e 2018 e de exclusão: artigos que não disponíveis na modalidade free e estudos secundários, artigos sem resumos disponíveis, sendo realizada no mês de Junho à Julho de 2018, tendo como amostra final 16 materiais. Diversos são os fatores apontados como causadores da ISC, dentre estes estão os intrínsecos e os extrínsecos. Pesquisas internacionais apontam incidência de infecção global de 3,4\%, já em 2017 investigações feitas no Brasil a incidência ocupa uma colocação de 3ำ lugar. Diante disso, concluímos que ao tomar medidas de controle simples essas infecções podem ser evitadas, assim a enfermagem é primordial na atuação desse controle.
\end{abstract}

Palavras Chave: Infecção da Ferida Cirúrgica, Cuidados de Enfermagem, Enfermagem, Cirurgia Geral, Prevenção.

\begin{abstract}
Surgical Site Infections (ISC) are diseases that affect patients submitted to some surgical procedure and represent $15 \%$ of hospital infections. The objective was to characterize the national and international scientific production on the subject, discussing interventions and nursing care from the perspective of ISC prevention. This is an integrative review based on a
\end{abstract}


bibliographic survey of 2275 materials in the databases: BDENF ; IBECS; LILACS; MEDLINE and SCIELO, from the inclusion criteria: full text articles, available and online in the vernacular, English or Spanish language, published between 2014 and 2018 and exclusion: articles that are not available in the free mode and secondary studies, articles without abstracts available, being carried out in the month of June to July 2018, having as final sample 16 materials. There are several factors that have been identified as causing ISC, among which are intrinsic and extrinsic. International research indicates a global infection incidence of 3.4\%, already in 2017 investigations done in Brazil the incidence occupies a placement of 3rd place. In view of this, we conclude that by taking simple control measures these infections can be avoided, so nursing is paramount in the performance.

Keywords: Surgical Wound Infection, Nursing Care, Nursing, General Surgery, Prevention.

\section{Introdução}

As Infecções de Sítio Cirúrgico (ISC's) são agravos que acometem pacientes que se submetem a algum procedimento operatório. Estas, porém representam complicações comuns, podendo representar $15 \%$ das infecções hospitalares. Tal incidência pode chegar a $60 \%$ durante o tempo de internação, revelando um número alarmante no ambiente hospitalar (RODRIGUES, et al., 2014).

Infecções Relacionada a Assistência à Saúde (IRAS) são consideradas uma das causas de ISC que mais afetam a recuperação do paciente em pós cirúrgico, o que gera maiores gastos com assistência, visto que um paciente quando evolui para esta condição, pode necessitar fazer o uso de antibiótico até ser submetido a um novo procedimento (SANTOS, et al., 2015).

A taxa de incidência de pacientes com infecção cirúrgica tem aumentado como resultado do contato direto durante cuidados em saúde. Apesar de existirem outros elementos que contribuem para o desenvolvimento da ISC, cabe destacar: idade, doenças crônicas, estado nutricional e etc. Além disso, o ambiente e a equipe multiprofissional também são fatores importantes no que se refere a transmissão de patógenos. Nessa perspectiva, reconhecendo que os profissionais da equipe de enfermagem mantêm uma maior proximidade com os pacientes, admite-se que tenham um papel primordial no controle dessa complicação (REIS, RODRIGUES, 2017).

Destaca-se, portanto, a importância do enfermeiro e sua atuação no controle e prevenção da ISC, no sentido de identificar e compreender os fatores de riscos, bem como o processo infeccioso no sítio cirúrgico, a fim de 
implementar medidas de promoção da saúde e rápida reabilitação (CARVALHO, et al., 2015).

Isto posto, objetivou-se caracterizar a produção científica nacional e internacional sobre o tema, discutindo intervenções e cuidados de enfermagem na perspectiva da prevenção da infecção do sítio cirúrgico (ISC).

\section{Procedimentos metodológicos}

Trata-se de uma Revisão Integrativa da literatura (RI) construída a partir de fontes secundárias, através de um levantamento de materiais bibliográficos no meio eletrônico. Esta vem sendo utilizada como um meio metodológico e também como um recurso sistemático que tem por objetivo reunir e sintetizar resultados de estudos enfocando um tema específico (MENDES; SILVEIRA; GALVÃO, 2008).

Segundo Mendes;Silveira;Galvão (2008) e Soares, et al., (2014) este método é estruturado em seis etapas: identificação do tema e seleção da hipótese ou questão de pesquisa; critérios para inclusão e exclusão de estudos, amostragem e busca na literatura; definição das informações; avaliação dos estudos incluídos na $\mathrm{RI}$; interpretação dos resultados $\mathrm{e}$ apresentação da revisão.

Foram estabelecidos como critérios de inclusão: artigos com texto completos, disponíveis e online na língua vernácula, inglesa ou espanhola, publicados entre 2014 e 2018, e que de fato se relacionem com o tema da presente revisão. Os critérios de exclusão foram: artigos que não estivessem disponíveis na modalidade free e estudos secundários, a exemplos de carta ao leitor, réplicas e duplicatas, editais, opiniões e comentários, teses, dissertações e artigos sem resumos disponíveis.

Para compor o corpus da pesquisa buscaram-se artigos indexados online nas bases de dados eletrônicas BDENF; LILACS; IBECS, como também, bases de dados como parte do portal PUBMED o qual engloba a MEDLINE e biblioteca digital ao qual engloba a SCIELO, nos meses de Junho e Julho de 2018. Foram utilizados os seguintes descritores: Infecção da Ferida Cirúrgica; 
Cuidados de Enfermagem; Enfermagem; Cirurgia Geral; Prevenção de Doenças.

Foi realizado o cruzamento dos descritores em Ciências da Saúde (DeCS) selecionados, utilizando o operador booleano "AND" da seguinte forma: Cirurgia Geral AND infecção de ferida cirúrgica AND Enfermagem; Infecção de ferida Cirúrgica AND prevenção de Doenças; Infecção da Ferida Cirúrgica AND Cuidados de Enfermagem; Infecção de Ferida Cirúrgica AND Enfermagem. Desta forma a população foi de 2.275 artigos indexados nas bases de dados consultadas a partir dos DECS, sendo a filtragem: 168 na base de dados BDENF; 165 na base IBECS; 266 na base LILACS, 1597 na base MEDLINE; 79 na base SCIELO. Foram excluídos 2269 artigos, permanecendo na amostra final 16 materiais. Tais estratégias de busca estão descritas na tabela 1 abaixo.

Tabela 1: Distribuição dos artigos encontrados, conforme as bases eletrônicas consultadas. Cuité/PB, 2018.

\begin{tabular}{|c|c|c|c|c|c|c|}
\hline \multirow{2}{*}{$\begin{array}{l}\text { DESCRITORES } \\
\text { CRUZADOS }\end{array}$} & \multicolumn{5}{|c|}{ ESTUDOS ENCONTRADOS } & \multirow[t]{2}{*}{ TOTAL } \\
\hline & BDENF & IBECS & LILACS & MEDLINE & SCIELO & \\
\hline $\begin{array}{lr}\text { Cirurgia } & \text { Geral } \\
\text { AND } & \text { Infecção } \\
\text { de } & \text { ferida } \\
\text { Cirúrgica } & \text { (IFC) } \\
\text { AND } & \\
\text { Enfermagem. }\end{array}$ & 81 & 19 & 111 & 227 & 41 & 479 \\
\hline $\begin{array}{l}\text { IFC AND } \\
\text { Prevenção de } \\
\text { Doencas }\end{array}$ & 36 & 104 & 0 & 1370 & 0 & 1510 \\
\hline $\begin{array}{l}\text { Doenças } \\
\text { IFC AND } \\
\text { Cuidados de } \\
\text { Enfermagem. }\end{array}$ & 16 & 14 & 127 & 0 & 04 & 161 \\
\hline $\begin{array}{l}\text { IFC AND } \\
\text { Enfermagem }\end{array}$ & 35 & 28 & 28 & 0 & 34 & 125 \\
\hline
\end{tabular}

FONTE: Dados da pesquisa 2018.

\section{$3 \quad$ Resultados}

Após filtragem e análise criteriosa dos artigos e considerando-se os critérios de exclusão, obteve-se um quantitativo de 16 materiais, uma vez que a maioria não apresentava respostas para o objetivo desta pesquisa. Permaneceram na 
amostra final, 03 artigos da BDENF; 03 da LILACS; 01 do IBECS; 02 MEDLINE e 07 da SCIELO. No quadro são apresentadas as bases de dados consultadas, título das referências identificadas com relevância para o estudo, bem como autor (es), periódico/ano de publicação.

Quadro 1. Apresentação dos artigos incluídos na RI.

\begin{tabular}{|c|c|c|c|}
\hline $\begin{array}{l}\text { BASE DE } \\
\text { DADOS }\end{array}$ & TÍTULO & $\begin{array}{c}\text { AUTORES } \\
\text { ANO }\end{array}$ & PERIÓDICO \\
\hline BDENF & $\begin{array}{l}\text { Fatores predisponentes para } \\
\text { infecção da ferida operatória } \\
\text { pós-cesárea: uma revisão } \\
\text { integrativa }\end{array}$ & $\begin{array}{l}\text { CARVALHO; } \\
\text { SOUZA; } \\
\text { MEDEIROS, } \\
2014\end{array}$ & $\begin{array}{l}\text { Fundam. care. } \\
\text { online }\end{array}$ \\
\hline SCIELO & $\begin{array}{l}\text { Avaliação de pacientes } \\
\text { quanto à infecção de sítio } \\
\text { cirúrgico, em um hospital } \\
\text { público de Belém-PA. }\end{array}$ & $\begin{array}{l}\text { RODRIGUES, } \\
\text { et al., } 2014\end{array}$ & $\begin{array}{l}\text { Revista } \\
\text { Paraense de } \\
\text { Medicina }\end{array}$ \\
\hline BDENF & $\begin{array}{l}\text { Fatores de risco de infecção } \\
\text { da ferida } \\
\text { operatória em neurocirurgia. }\end{array}$ & $\begin{array}{l}\text { BELLUSSI, et } \\
\text { al., } 2015\end{array}$ & $\begin{array}{ll}\text { Acta } & \text { Paul } \\
\text { Enferm } & \end{array}$ \\
\hline BDENF & $\begin{array}{l}\text { Incidência e fatores de risco } \\
\text { de infecção de sítio cirúrgico: } \\
\text { revisão integrativa }\end{array}$ & $\begin{array}{l}\text { SANTOS, et } \\
\text { al., } 2015\end{array}$ & $\begin{array}{l}\text { Itinerairus } \\
\text { Reflections }\end{array}$ \\
\hline LILACS & $\begin{array}{l}\text { Tempo de internação pré- } \\
\text { operatório: um fator de risco } \\
\text { para reduzir a infecc,ão } \\
\text { cirúrgica em fraturas de } \\
\text { fêmur. }\end{array}$ & $\begin{array}{l}\text { PEREIRA, } \\
\text { REZENDE, } \\
\text { COUTO, } 2015\end{array}$ & $\begin{array}{l}\text { Rev. } \\
\text { Ortop }\end{array}$ \\
\hline SCIELO & $\begin{array}{l}\text { Validação de checklist } \\
\text { cirúrgico para } \\
\text { prevenção de infecção de } \\
\text { sítio cirúrgico. }\end{array}$ & $\begin{array}{l}\text { ROSCANI, et } \\
\text { al., } 2015\end{array}$ & $\begin{array}{l}\text { Acta } \\
\text { Enferm }\end{array}$ \\
\hline SCIELO & $\begin{array}{l}\text { Readmissão por infecção do } \\
\text { sítio cirúrgico ortopédico: } \\
\text { uma revisão integrativa. }\end{array}$ & $\begin{array}{l}\text { TORRES, et } \\
\text { al., } 2015\end{array}$ & $\begin{array}{l}\text { Rev Esc } \\
\text { Enferm USP }\end{array}$ \\
\hline SCIELO & $\begin{array}{l}\text { Conhecimento dos } \\
\text { profissionais de enfermagem } \\
\text { sobre fatores de risco } \\
\text { relacionados à infecção de } \\
\text { sítio cirúrgico. }\end{array}$ & $\begin{array}{l}\text { CARVALHO, } \\
\text { et al., } 2015\end{array}$ & Revista. Interd \\
\hline IBECS & $\begin{array}{l}\text { Indicadores de processo para } \\
\text { prevenção da infecção do } \\
\text { sítio cirúrgico sob a ótica da }\end{array}$ & $\begin{array}{l}\text { GEBRIM, } \\
\text { al., } 2016\end{array}$ & $\begin{array}{l}\text { Revista } \\
\text { electronica } \\
\text { trimestral de }\end{array}$ \\
\hline
\end{tabular}




\begin{tabular}{|c|c|c|c|}
\hline & segurança do paciente. & & enfermeria \\
\hline MEDLINE & $\begin{array}{l}\text { Prevenção de infecção do } \\
\text { sítio cirúrgico: uma prioridade } \\
\text { global. }\end{array}$ & $\begin{array}{l}\text { ABBAS, } \\
\text { PITTET, } 2016\end{array}$ & $\begin{array}{l}\text { Journal of } \\
\text { Hospital } \\
\text { Infection/Elsevi } \\
\text { er }\end{array}$ \\
\hline SCIELO & $\begin{array}{l}\text { O Enfermeiro e a prevenção } \\
\text { das infecções } \\
\text { do sítio cirúrgico. }\end{array}$ & $\begin{array}{l}\text { ROCHA, } \\
\text { LAGES, } 2016\end{array}$ & $\begin{array}{l}\text { Cadernos } \\
\text { UniFOA }\end{array}$ \\
\hline LILACS & $\begin{array}{l}\text { Infecção de sítio cirúrgico } \\
\text { pós-alta: ocorrência e } \\
\text { caracterização de egressos } \\
\text { de cirurgia geral. }\end{array}$ & $\begin{array}{l}\text { REIS, } \\
\text { RODRIGUES, } \\
2017\end{array}$ & $\begin{array}{l}\text { Cogitare } \\
\text { Enferm }\end{array}$ \\
\hline LILACS & $\begin{array}{l}\text { Incidência e fatores de risco } \\
\text { para infecção de sítio } \\
\text { cirúrgico em cirurgias gerais. }\end{array}$ & $\begin{array}{l}\text { CARVALHO, } \\
\text { et al., } 2017\end{array}$ & $\begin{array}{l}\text { Rev latino } \\
\text { americana de } \\
\text { enfermagem }\end{array}$ \\
\hline MEDLINE & $\begin{array}{l}\text { Introduction to the Centers for } \\
\text { Disease Control } \\
\text { and Prevention and } \\
\text { Healthcare Infection } \\
\text { Control Practices Advisory } \\
\text { Committee Guideline } \\
\text { for Prevention of Surgical Site } \\
\text { Infection: } \\
\text { Prosthetic Joint Arthroplasty } \\
\text { Section. }\end{array}$ & $\begin{array}{l}\text { SEGRETI, et } \\
\text { al., } 2017\end{array}$ & $\begin{array}{l}\text { Surgical } \\
\text { Infections }\end{array}$ \\
\hline SCIELO & $\begin{array}{l}\text { Efeitos do banho pré- } \\
\text { operatório na prevenção de } \\
\text { infecção cirúrgica: } \\
\text { estudo clínico piloto. }\end{array}$ & $\begin{array}{l}\text { FRANCO, et } \\
\text { al., } 2017\end{array}$ & $\begin{array}{l}\text { Rev } \\
\text { Enferm }\end{array}$ \\
\hline SCIELO & $\begin{array}{l}\text { Pré-operatório de cirurgias } \\
\text { potencialmente } \\
\text { contaminadas: fatores de } \\
\text { risco para infecção do sítio } \\
\text { cirúrgico. }\end{array}$ & $\begin{array}{l}\text { MARTINS, et } \\
\text { al., } 2017\end{array}$ & $\begin{array}{l}\text { Acta } \\
\text { Enferm }\end{array}$ \\
\hline
\end{tabular}

FONTE: Dados da pesquisa, 2018.

Em relação a análise das bases de dados, verificou-se que estudos que abordavam a temática, foram localizados em maior número de publicações na base de dado SCIELO totalizando 7 artigos (43,75\%). Dessa forma, observa-se quanto ao ano de publicação que dos 16 estudos, 2 (12,5\%) foram publicados em 2014; 6 (37,5\%) no ano de 2015, 3 em 2016 (18,75\%) e $5(31,25 \%)$ em 2017, observando-se assim um maior número de estudos publicados no ano de 2015. O períodico identificado com maior número de publicações foi a Acta Paulista de Enfermagem com 3 publicações (18,75\%). 
Nos estudos analisados, 5 (31,25\%) apontam informações referentes aos fatores de risco para ISC; 5 (31,25\%) sobre formas de prevenção da ISC; 4 (25\%) sobre incidência/prevalência dos casos de ISC e apenas 2 (12,5\%) sobre o conhecimento dos profissionais de enfermagem e os cuidados de enfermagem na prevenção da ISC.

Para melhor compreender a temática investigada, considerou-se relevante fazer uma análise pormenorizada dos materiais disponíveis na íntegra nas bases de dados consultadas. Assim, foi construído um quadro apresentando o título, os objetivos e principais resultados dos materiais selecionados, conforme segue:

Quadro 2. Apresentação da síntese de artigos incluídos na RI.

\begin{tabular}{|c|c|c|}
\hline TÍTULO & OBJETIVO & RESULTADOS \\
\hline $\begin{array}{l}\text { Fatores } \\
\text { predisponentes para } \\
\text { infecção da ferida } \\
\text { operatória pós- } \\
\text { cesárea: uma revisão } \\
\text { integrativa. }\end{array}$ & $\begin{array}{l}\text { Investigar, na literatura } \\
\text { pertinente ao tema, os } \\
\text { fatores predisponentes } \\
\text { para infecção da ferida } \\
\text { operatória pós-cesárea, } \\
\text { nos últimos cinco anos. }\end{array}$ & $\begin{array}{l}\text { Obesidade, tabagismo, } \\
\text { trabalho de parto } \\
\text { prolongado, ruptura da } \\
\text { membrana, baixo status } \\
\text { socioeconômico, } \\
\text { doenças } \\
\text { vasculares, uso dênico- } \\
\text { corticoide, estresse, } \\
\text { nutrição, hipotermia, } \\
\text { anestesia, toques } \\
\text { vaginais, são fatores } \\
\text { agravantes de ISC. }\end{array}$ \\
\hline $\begin{array}{llr}\text { Avaliação } & & \text { de } \\
\text { pacientes } & \text { quanto à } \\
\text { infecção } & \text { de } & \text { sítio } \\
\text { cirúrgico, em } & \text { um } \\
\text { hospital público } & \text { de } \\
\text { Belém-pa. } & \end{array}$ & $\begin{array}{l}\text { Avaliar os pacientes } \\
\text { admitidos no serviço de } \\
\text { cirurgia geral do hospital } \\
\text { FHCGV, quanto ao } \\
\text { comportamento da } \\
\text { infecção de sítio cirúrgico. }\end{array}$ & $\begin{array}{l}\text { Tabagismo, obesidade, } \\
\text { desnutrição, HAS, DM, } \\
\text { Cardiopatias, } \\
\text { imunossupressão, } \\
\text { idade, sexo, porte } \\
\text { cirurgico, classificação } \\
\text { ASA, classificação } \\
\text { cirurgica, } \\
\text { condicionantes são } \\
\text { ISC. }\end{array}$ \\
\hline $\begin{array}{lr}\text { Fatores de risco de } \\
\text { infecção da } & \text { ferida } \\
\text { operatória } & \text { em } \\
\text { neurocirurgia. } & \end{array}$ & $\begin{array}{lr}\text { Analisar os fatores de } \\
\text { risco de infecção da ferida } \\
\text { operatória } & \text { em } \\
\text { neurocirurgia } & \end{array}$ & $\begin{array}{l}\text { A ISC apresentou maior } \\
\text { prevalência em idosos, } \\
\text { hipertensos e diabéticos, } \\
\text { submetidos a cirugia de } \\
15 \text { á } 400 \text { min, cirurgias } \\
\text { de porte I, com tempo } \\
\text { de internação > que } 6 \\
\text { dias. }\end{array}$ \\
\hline
\end{tabular}




\begin{tabular}{|c|c|c|}
\hline $\begin{array}{l}\text { Incidência e fatores } \\
\text { de risco de infecção } \\
\text { de sítio cirúrgico: } \\
\text { revisão integrativa. }\end{array}$ & $\begin{array}{l}\text { Determinar, baseado nas } \\
\text { produções cientificas } \\
\text { publicadas nos últimos } 10 \\
\text { anos, a incidência de } \\
\text { infecção do sitio cirúrgico } \\
\text { (ISC) e dos fatores de } \\
\text { risco relacionados a ela. }\end{array}$ & $\begin{array}{l}\text { Pacientes > de } 30 \text { anos } \\
\text { do sexo masculino, } \\
\text { apresentam maiores } \\
\text { chances de ISC. Os } \\
\text { patogenos identificados } \\
\text { de > prevalência foram: } \\
\text { Pseudomonas A. } \\
\text { Escherichia c; Klebsiella } \\
\text { P, identificadas em > } \\
\text { frequência nas cirurgias } \\
\text { de TGI, com tempo } \\
\text { cirurgico > } 1 \mathrm{~h} \text { e tempo } \\
\text { de internação }>2 \text { dias. }\end{array}$ \\
\hline $\begin{array}{l}\text { Tempo de internação } \\
\text { pré-operatório: um } \\
\text { fator de risco para } \\
\text { reduzir a infecção } \\
\text { cirúrgica em fraturas } \\
\text { de fêmur. }\end{array}$ & $\begin{array}{l}\text { Analisar as infecções de } \\
\text { sítio cirúrgico em } \\
\text { pacientes submetidos a } \\
\text { cirurgias limpas para } \\
\text { correcção de fraturas de } \\
\text { fêmur. }\end{array}$ & $\begin{array}{l}\text { Tempo de internação > } \\
\text { que } 4 \text { dias, torna o risco } \\
\text { esperado de ISC } 3 \\
\text { vezes maior, pacientes } \\
\text { com classificação ASA I, } \\
\text { com procedimentos de } \\
\text { cirurgia eletiva, são mais } \\
\text { acometidos } \\
\text { patogenos: } \\
\text { stapylococcus } \\
\text { acinetobacter baumanii. }\end{array}$ \\
\hline $\begin{array}{lr}\text { Validação } & \text { de } \\
\text { checklist cirúrgico } \\
\text { para prevenção de } \\
\text { infecção de sítio } \\
\text { cirúrgico. }\end{array}$ & $\begin{array}{l}\text { Construir e validar um } \\
\text { instrumento de verificação } \\
\text { de segurança cirúrgica } \\
\text { para aumentar a } \\
\text { segurança do paciente e } \\
\text { para auxiliar na prevenção } \\
\text { de infecção de sítio } \\
\text { cirúrgico }\end{array}$ & $\begin{array}{l}\text { Cuidados no } \\
\text { transoperatório, como } \\
\text { tomar banho com } \\
\text { substâncias } \\
\text { antissépticas no pré- } \\
\text { operatório, considerar a } \\
\text { avaliação/ segurança do } \\
\text { paciente, uso da } \\
\text { profilaxia são formas de } \\
\text { prevenir a ISC. }\end{array}$ \\
\hline $\begin{array}{lr}\text { Readmissão } & \text { por } \\
\text { infecção do sítio } \\
\text { cirúrgico ortopédico: } \\
\text { uma } \\
\text { integrativa. }\end{array}$ & $\begin{array}{l}\text { Caracterizar a readmissão } \\
\text { por infecção do sítio } \\
\text { cirúrgico ortopédico nos } \\
\text { últimos cinco anos. }\end{array}$ & $\begin{array}{l}\text { Fator de readmissão foi } \\
\text { a reoperação por ISC, } \\
\text { nos primeiros } 30 \text { dias, } \\
\text { gerando tempo de } \\
\text { internação > de } 5 \text { á } 9 \\
\text { dias. Falta de educação } \\
\text { em saúde para com o } \\
\text { cuidados da ferida } \\
\text { operatória também são } \\
\text { causas de ISC. }\end{array}$ \\
\hline
\end{tabular}




\begin{tabular}{|c|c|c|}
\hline $\begin{array}{lr}\text { Conhecimento } & \text { dos } \\
\text { profissionais } & \text { de } \\
\text { enfermagem } & \text { sobre } \\
\text { fatores de } & \text { risco } \\
\text { relacionados } & \text { à } \\
\text { infecção de } & \text { sítio } \\
\text { cirúrgico. } & \\
\end{array}$ & $\begin{array}{l}\text { Avaliar o conhecimento } \\
\text { dos profissionais de } \\
\text { enfermagem sobre fatores } \\
\text { de risco relacionados a } \\
\text { Infecção de Sítio } \\
\text { Cirúrgico. }\end{array}$ & $\begin{array}{l}\text { Qualificação } \\
\text { profissional, intervenção } \\
\text { da CCIH, uso de EPI's, } \\
\text { práticas antissépticas e } \\
\text { assépticas, são } \\
\text { considerados formas de } \\
\text { prevenção da ISC. }\end{array}$ \\
\hline $\begin{array}{lr}\text { Indicadores } & \text { de } \\
\text { processo para } & \text { a } \\
\text { prevenção } & \text { da } \\
\text { infecção do sítio } \\
\text { cirúrgico do ponto de } \\
\text { vista do paciente }\end{array}$ & $\begin{array}{l}\text { Avaliar os indicadores } \\
\text { de processo para a } \\
\text { prevenção da infecção } \\
\text { do sítio cirúrgico em } \\
\text { cirurgias limpas em um } \\
\text { hospital universitário do } \\
\text { Centro-Oeste brasileiro . }\end{array}$ & $\begin{array}{l}\text { Indicadores de processo } \\
\text { para a cirurgia segura, } \\
\text { como: tempo de } \\
\text { internação, tricotomia } \\
\text { correta, profilaxia } \\
\text { antimicrobiana,antisseps } \\
\text { ia do campo operatório, } \\
\text { controle glicêmico em } \\
\text { diabeticos, registro de } \\
\text { caixas cirurgicas, foram } \\
\text { considerado inadequado } \\
(64,6 \%) \text {. }\end{array}$ \\
\hline \begin{tabular}{lr} 
Prevenção & de \\
infecção do sítio \\
cirúrgico: & \multicolumn{1}{c}{ uma } \\
prioridade global.
\end{tabular} & $\begin{array}{l}\text { artigo de Choi e } \\
\text { colaboradores relata as } \\
\text { tendências nas taxas de } \\
\text { ISC após gastrectomia e } \\
\text { após artroplastia total de } \\
\text { quadril e joelho total após } \\
\text { a introdução do KONIS. }\end{array}$ & $\begin{array}{l}\text { Há uma } r \text { maior } \\
\text { prevalência ISC em } \\
\text { países pobres, } \\
\text { prevenção e medidas de } \\
\text { controle, como técnicas } \\
\text { antissépticas } \\
\text { assépticas durante } \\
\text { procedimentos } \\
\text { invasivos, são de } \\
\text { primodial importância, } \\
\text { cuidados antes, durante } \\
\text { e após o procedimento } \\
\text { são a consideradas } \\
\text { medidas de profilaxia. }\end{array}$ \\
\hline $\begin{array}{lr}\text { O Enfermeiro e a } \\
\text { prevenção } & \text { das } \\
\text { infecções } & \\
\text { do sítio cirúrgico. } & \end{array}$ & $\begin{array}{l}\text { Descrever as principais } \\
\text { causas de infecção no } \\
\text { sítio cirúrgico; pesquisar a } \\
\text { atuação da Comissão de } \\
\text { Controle de Infecção } \\
\text { Hospitalar (CCIH) na } \\
\text { prevenção das Infecções } \\
\text { no sítio cirúrgico e; } \\
\text { investigar as } \\
\text { condutas de Enfermagem } \\
\text { para a prevenção das } \\
\text { Infecções no sítio } \\
\text { cirúrgico. }\end{array}$ & $\begin{array}{l}\text { Agentes etiológicos, } \\
\text { paciente, morbidade, } \\
\text { procedimento cirúrgico, } \\
\text { uso inadequado de } \\
\text { medicamentos, baixo } \\
\text { nível de educação, falta } \\
\text { de profissionais de } \\
\text { enfermagem } \\
\text { qualificados e medidas } \\
\text { de prevenção e controle } \\
\text { inadequadas, predispõe } \\
\text { pacientes a ISC. }\end{array}$ \\
\hline
\end{tabular}




\begin{tabular}{|c|c|c|}
\hline $\begin{array}{lrr}\text { Infecção de } & \text { sítio } \\
\text { cirúrgico pós-alta: } \\
\text { ocorrência } & \text { e } \\
\text { caracterização } & \text { de } \\
\text { egressos de cirurgia } \\
\text { geral. }\end{array}$ & $\begin{array}{l}\text { Investigar a ocorrência de } \\
\text { infecção de sítio cirúrgico } \\
\text { e descrever as } \\
\text { características dos casos } \\
\text { de pacientes } \\
\text { seguimento pós-alta de } \\
\text { Cirurgia Geral, em } \\
\text { hospital de ensino do } \\
\text { Distrito Federal, Brasil. }\end{array}$ & $\begin{array}{l}\text { Cirugias gerais, cirurgias } \\
\text { limpas, são apontadas } \\
\text { com uma alta } \\
\text { prevalência de ISC, } \\
\text { pacientes > de } 60 \text { anos, } \\
\text { período de operação } \\
\text { cirúrgica e o diagnóstico } \\
\text { de ISC, também } \\
\text { contribuem para agravos } \\
\text { a saúde do paciente } \\
\text { cirurgico. }\end{array}$ \\
\hline $\begin{array}{l}\text { Incidência e } \\
\text { de ratores } \\
\text { infecção de de sítio } \\
\text { cirúrgico em cirurgias } \\
\text { gerais. }\end{array}$ & $\begin{array}{l}\text { Estimar a incidência de } \\
\text { ISC em cirurgias gerais de } \\
\text { um hospital brasileiro de } \\
\text { grande porte, identificando } \\
\text { fatores de risco e } \\
\text { microrganismos } \\
\text { prevalentes. }\end{array}$ & $\begin{array}{l}\text { Procedimentos } \\
\text { cirurgicos (PC) } \\
\text { realizados em pessoas } \\
\text { do sexo feminino com } \\
\text { idade > de } 54 \text { anos, com } \\
\text { tempo de PC > 1,6 h, } \\
\text { apresentam ISC após } \\
\text { período de internação > } \\
\text { que } 24 \text { hrs, em } \\
\text { pacientes clasificados } \\
\text { como ASA I, após PC de } \\
\text { cirurgia limpa, eletiva, } \\
\text { sem uso de anestesia } \\
\text { geral ou uso de } \\
\text { implante, causada por } \\
\text { patógenos: } \\
\text { stapyloccocus aureus e } \\
\text { Escherichia coli. }\end{array}$ \\
\hline $\begin{array}{l}\text { Introdução } \\
\text { Centros de Controle } \\
\text { e Prevenção de } \\
\text { Doenças e Infecção } \\
\text { em Saúde } \\
\text { Diretriz do Comitê } \\
\text { Consultivo de } \\
\text { Práticas de Controle } \\
\text { para Prevenção da } \\
\text { Infecção do Local } \\
\text { Cirúrgico: Seção de } \\
\text { Artroplastia da da drática. } \\
\text { Articulação Protética. }\end{array}$ & 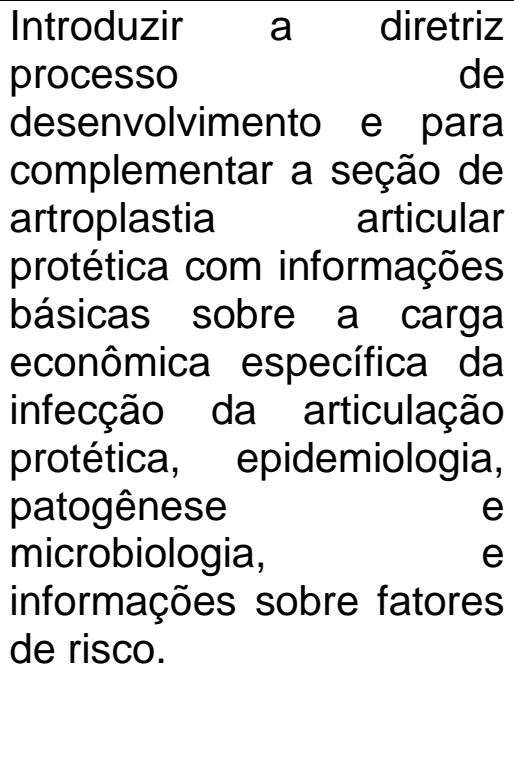 & $\begin{array}{l}\text { ISC é considerada de } \\
\text { alta incidência, } \\
\text { acomentendo pacientes } \\
\text { com procedimentos de } \\
\text { incisão profunda e } \\
\text { orgãos, procedimentos } \\
\text { invasivos como cateres } \\
\text { e sondas, ao qual } \\
\text { surgem organismos } \\
\text { multiresistentes } \\
\text { diversas a } \\
\text { ameaçando a vida. } \\
\text { Fatores de risco pre- } \\
\text { operatório e pós- } \\
\text { operatório também } \\
\text { contribue para a ISC. }\end{array}$ \\
\hline $\begin{array}{ll}\text { Efeitos do banho pré- } \\
\text { operatório } & \text { na } \\
\text { prevenção } & \text { de } \\
\text { infecção cirúrgica: } & \end{array}$ & $\begin{array}{l}\text { Avaliar o efeito do banho } \\
\text { pré-operatório na } \\
\text { prevenção de ISC, } \\
\text { utilizando duas soluções }\end{array}$ & $\begin{array}{l}\text { Banho pré operatório } \\
\text { com grupos de } \\
\text { intervenção PVPI } 10 \% \text {; } \\
\text { clorexidina e sabão não }\end{array}$ \\
\hline
\end{tabular}




\begin{tabular}{|c|c|c|}
\hline estudo clínico piloto. & $\begin{array}{l}\text { antissépticas - gluconato } \\
\text { de clorexidina a } 4 \% \text { e } \\
\text { PVPI degermante a } 10 \%- \\
\text { e um sabão sem } \\
\text { antisséptico, em pacientes } \\
\text { submetidos a cirurgia } \\
\text { eletiva de artroplastia do } \\
\text { quadril. }\end{array}$ & $\begin{array}{l}\text { houveram maiores } \\
\text { diferenças estatísticas. } \\
\text { Intervenção realizada } \\
\text { em pacientes do sexo } \\
\text { mascuino, ASA II, } \\
\text { cirurgias limpas, como } \\
\text { uso de } \\
\text { quimioprofilaxia/cefalozi } \\
\text { na durante } 24 \text { hr, o } \\
\text { tempo do banho até a } \\
\text { incisão foi de } 81 \text { à } 306 \\
\text { min, com tempo de } \\
\text { internação de } 2 \text { à } 8 \text { dias. }\end{array}$ \\
\hline $\begin{array}{ll}\text { Pré-operatório } & \text { de } \\
\text { cirurgias } & \\
\text { potencialmente } & \\
\text { contaminadas: } & \\
\text { fatores de risco para } \\
\text { infecção do sítio } \\
\text { cirúrgico. }\end{array}$ & $\begin{array}{l}\text { Associar os fatores de } \\
\text { risco do período pré- } \\
\text { operatório, de cirurgias } \\
\text { potencialmente } \\
\text { contaminadas, realizadas } \\
\text { em um hospital escola da } \\
\text { região Sul do Brasil, com } \\
\text { a ocorrência da infecção } \\
\text { do sitio cirúrgico no } \\
\text { período pós-operatório } \\
\text { hospitalar e em domicilio. }\end{array}$ & $\begin{array}{l}\text { Pacientes obesos, com } \\
\text { grau de dependência } \\
\text { maior que } 50 \% \text { o, } \\
\text { apresentando HAS, } \\
\text { fumante, etilista, com } \\
\text { tempo de internação > } \\
\text { que } 6 \text { dias, solitários, } \\
\text { são fatores modificavéis } \\
\text { e não modificavéis que } \\
\text { contribuem para ISC no } \\
\text { pré e pós-operatório. }\end{array}$ \\
\hline
\end{tabular}

FONTE: Dados da pesquisa, 2018.

A respeito dos fatores de risco para a ISC, os autores citam diversas condições que predispõem a Infecção da Ferida Cirúrgica (IFC) sendo estes podendo ser modificáveis e não modificáveis, tornando-se importante sua identificação desde a marcação do procedimento cirúrgico até o pós- operatório (MARTINS, 2017; CARVALHO, et al., 2017; SANTOS, et al., 2015; CARVALHO; SOUZA; MEDEIROS, 2014).

A obesidade e Hipertensão Arterial Sistêmica (HAS) é vista em 3 $(18,75 \%)$ estudos (CARVALHO; SOUZA; MEDEIROS, 2014; RODRIGUES, et al., 2014; BELLUSI, et al., 2015); outras causas são: o tabagismo identificado em 2 (12,5\%) (CARVALHO; SOUZA; MEDEIROS, 2014; RODRIGUES, et al., 2014); doenças cardiovasculares citadas também em 2 pesquisas (12,5\%) (CARVALHO; SOUZA; MEDEIROS, 2014; RODRIGUES, et al., 2014); uso de corticosteroides em 1 dos estudos (6,5\%) (CARVALHO, 2014); procedimentos invasivos como sondas vesicais e cateteres identificados em 2 (12,5) (REIS, RODRIGUES, 2017; ABBAS, 2016); desnutrição em 1 (6,5\%) (PEREIRA; 
REZENDE; COUTO, 2015); Diabetes Mellitos (DM) em 2 pesquisas (12,5\%) (RODRIGUES, et al., 2014; CARVALHO; SOUZA; MEDEIROS, 2014); nível educacional baixo apresentado em 3 investigações (18,5\%) (CARVALHO, 2015; ABBAS, 2016; ROCHA; LAGES, 2016); em dois (12,5\%) o uso inadequado de medicações profiláticas (SANTOS, et al.,2015; ROSCANI, et al., 2015); em 1 (6,5\%) estresse (CARVALHO; SOUZA; MEDEIROS, 2014); em 1 (6,5\%) imunossupressão (RODRIGUES, et al., 2014); em 1 (6,5\%) hipotermia (CARVALHO; SOUZA; MEDEIROS, 2014) e por fim 1 estudo (6,5\%) que cita a anestesia realizada (FRANCO, et al., 2017).

Os artigos trouxeram ainda informações sobre a incidência da IFC e mostraram que a idade e o sexo são também condições importantes a serem observadas no que se refere à prevalência deste problema de saúde. Identificou-se nos estudos analisados que há uma controvérsia quanto a incidência da IFC em pessoas do sexo masculino e feminino. Assim, identificou-se em estudos de dois autores (12,5\%) que a incidência é maior em homens (REIS; RODRIGUES, et al., 2017; BELUSSI, et al., 2015), já em 2 $(12,5 \%)$ prevalece o sexo feminino (SEGRETI, et al., 2017; CARVALHO, et al., 2017). No tocante à idade, sua incidência é contestável, visto que duas investigações mostraram maior números de casos em pessoas $>$ de 54 anos, outro > 30 anos e por fim > que 60 anos (REIS; RODRIGUES, 2017; CARVALHO, et al., 2017; SEGRETI, et al., 2017).

Ainda sobre incidência e prevalência dos casos, estudos feitos por Franco et al., (2017); Pereira; Rezende; Couto, et al., (2015) e Carvalho et al., (2017) mostraram que pacientes com classificação ASA I apresentaram maior índice de infecção, seguidos de paciente classificados como ASA II. Quanto ao uso de anestesia, verifica-se que a IFO é mais prevalente em indivíduos que se submeteram a não anestesia geral, cirurgia limpa, eletiva, seguidas de gerais, com incisões profundas ou de órgãos.

O tempo de internação e o tempo cirúrgico são também apontados como fatores que podem levar a ISC. Porém questiona-se esse apontamento, visto que para Belussi, et al., (2015) e Franco, et al., (2017) cirurgias com duração de 81 a 306 minutos representam fatores de riscos para a ISC. Já Santos, et al., (2015) informa que um tempo superior a 1 hora e 60 min é considerado um 
facilitador em potencial de ISC. No que se refere ao tempo de internação, Franco, et al., (2017); e Martins, et al., (2017) expõem um tempo > que 6 dias, porém, trata-se de uma discussão ainda inconclusiva, visto que em outro estudo feito por Santos, et al., (2015); Carvalho, et al., (2017) e Franco, et al., (2017) observa-se um período > que 24 h; já em outro feito por Pereira; Rezende; Couto, (2015) mostra um período > que 4 dias e por fim investigações feitas por Bellussi et al., (2015) e Torres, et al., (2015) um tempo de 2 a 8 dias.

No que se diz respeito aos patógenos, em mais da metade dos estudos, não havia dados sobre os microorganismos mais prevalentes e em três trabalhos (18,75\%), os microorganismos mais prevalente foram: Stapylococcus aureus, seguido de Escherichia coli, como também, Pseudomonas aeruginosa; Klebsiella pneumoniae e Acinetobater Baumani, dentre outros, porém apresentados aqui os mais prevalentes (SANTOS, et al., 2015; PEREIRA; REZENDE; COUTO, 2015; CARVALHO, et al., 2017).

A respeito das formas de prevenção da ISC, são citados o banho préoperatório, quimioprofilaxia até as 24 horas após procedimento cirúrgico, o uso de Equipamentos de Proteção Individual (EPI's) por profissionais, técnicas assépticas e antissépticas e os protocolos de segurança do paciente como formas de prevenir a IFC (FRANCO, et al., 2017; GEBRIM, et al., 2016; CARVALHO, et al., 2015; ROSCANI, et al., 2015).

\section{Discussão}

As ISC's estão relacionadas a procedimentos cirúrgicos, podendo associaremse ou não colocação de implantes. Nessa perspectiva, sugerem-se critérios para a identificação da ISC. Quando superficial ou envolve o tecido subcutâneo, pode ser identificada a partir do primeiro dia até ao $30^{\circ}$ dia após 0 procedimento, considera-se os seguintes requisitos: drenagem purulenta; cultura positiva ou algum sinal flogístico. Quando incisão profunda, a ISC pode ser identificada até 90 dias após o procedimento, principalmente aqueles com implantes. Para o diagnóstico é preciso a presença de pelo menos um dos critérios: drenagem purulenta; febre > que $38^{\circ} \mathrm{C}$; abcesso ou deiscência. Quando incisões de cavidades ou órgãos, o resultado de cultura positiva de 
órgão/cavidade; abcesso identificado em reoperação ou exame anatopatológico diagnosticam a infecção (BRASIL, 2017; SEGRETI, et al., 2017).

Diante dos achados observa-se que a ISC ainda é considerada nos dias atuais um risco para os pacientes que se submetem a procedimentos operatórios. No estudo de por Carvalho, et al., (2017), constatou-se que a incidência global é de 3,4\% em países desenvolvidos como os EUA; já em investigação desenvolvida no Brasil (2017), o país ocupa o 3ํ lugar no panorama de ISC, sendo influenciadas por IRAS (perfazendo um percentual de $14 \%$ a $16 \%$ encontrados em pacientes ainda hospitalizados, podendo $60 \%$ dos casos serem evitados).

O estudo de Reis; Rodrigues (2017) aponta que normalmente a assistência ao paciente cirúrgico acontece apenas no período pré-operatório imediato e mediado, sendo a assistência negligenciada no pós alta por outras redes de assistência a saúde, como as Unidades Básicas de Saúde (UBS), o que aumenta a probabilidade de ISC. Dentre os fatores contribuintes, cabe citar o déficit de ações voltadas à educação em saúde, influenciando no surgimento de complicações clínicas, na recuperação e gerando uma reabilitação tardia, que ocasiona o retorno ao hospital para a resolução de casos de infecção pósoperatória domiciliar (BELLUSSI, et al., 2015).

Além destes eventos, ABBAS (2016) e Carvalho; Souza; Medeiros (2014) expõem outras dificuldades que contribuem para causar a ISC, podendo ser esta condição influenciada pelo contexto social do paciente, entraves inerentes à distância entre domicílio e instituição e questões socioeconômicas. Assim, enfatiza-se a importância da avaliação da ISC no 30 dia após a cirurgia ou até 1 ano após o procedimento, principalmente em casos de implantes, visto que a ISC pode se manifestar durante este período (TORRES, et al., 2015; RODRIGUES, et al., 2014).

Condições relacionadas a fatores predisponentes para ISC são evidenciadas nos estudos de Martins, et al., (2017), Carvalho; Souza; Medeiros (2014) e Santos, et al., (2015), a exemplo de: condições clínicas, idade avançada, sexo, situação de moradia, obesidade, desnutrição, 
imunossupressão, tabagismo, etilismo, medicações, grau de dependência, doenças associadas (HAS e DM) e o fato de morar sozinho.

Outros fatores também contribuem para a ocorrência de ISC, podendo relacionarem-se ao ambiente/equipe de saúde, ao hospedeiro/paciente e ao agente etiológico (ROCHA; LAGES, 2016). Alguns fatores específicos são: o tempo de internação pré-operatória, a classificação do Procedimento Cirúrgico (PC) (Se cirurgia limpa, potencialmente contaminada, contamina ou infectada), o porte (I, II, III, IV, V), duração (tempo relacionado a maior exposição do sítio cirúrgico à patógenos e quebra da técnica asséptica) tipo do PC (se eletiva, de emergência e etc.), além de complicações pós-operatórias como: deiscência da ferida, infecção no trato urinário e choque séptico, fatores estes que podem ser amenizados a partir da identificação dos riscos, da anamnese e exame físico (FRANCO, et al., 2017).

No que se refere a quebra da técnica asséptica como fator de risco para IFC a nível hospitalar, investigações evidenciam o Staphylococcus aureus como sendo o microorganismo de maior prevalência, seguido da $E$. coli, sendo a primeira encontrada em instrumentais cirúrgicos, aparelhos, móveis da sala cirúrgica, prótese de implantes, materiais incorretamente esterilizados. Já a E.coli está presente principalmente em peles mal higienizadas. Tais fatores podem, portanto, ser evitados se respeitado o rigor da técnica asséptica, priorizando uma correta esterilização, bem como uma correta paramentação e antissepsia da pele (PEREIRA; REZENDE; COUTO, 2015).

Algumas medidas preventivas no período pré, intra e pós-operatório são citadas como formas de prevenção da IFC, a exemplo do banho pré-operatório. Porém, no estudo de Franco (2017) ao serem testadas algumas substâncias a fim de evidenciar seu impacto na prevalência da ISC, constatou-se que o banho com clorexidina a $4 \%$, sabão sem antisséptico e PVPI a $10 \%$ não ocasionaram maiores repercussões (BRASIL, 2017).

Há ainda outras medidas pré-operatórias, como o uso de pomada de mupirocina em determinados PC de pacientes com vias nasais colonizadas por Staphylococcus aureus; antibiótico endovenoso para profilaxia oral com início de 30 a 60 minutos antes da incisão cirúrgica até 24 horas após o procedimento; não remoção dos pêlos; preparação do sítio cirúrgico com 
soluções antissépticas adequadas; degermação cirúrgica correta com uso de técnica e antisséptico. Quanto às intra operatórias estão: apoio nutricional a pacientes desnutridos, a não interrupção de imunossupressores, uso de oxigenação em pacientes submetidos a cirurgia geral, manutenção da temperatura (controle da hipotermia e hipertermia), manutenção da normovolemia e uso de dispositivos de proteção de feridas e curativo simples (BRASIL, 2017).

A coordenação, supervisão, instrução e verificação, quanto a montagem da sala cirúrgica, antes do início do procedimento, a degermação da equipe cirúrgica e do paciente, a retirada de objetos pessoais (relógios, cordões, anéis, pulseiras), bem como a inspeção do material cirúrgico quanto a validade e correta esterilização, são formas de prevenção, todas evitáveis, que dependem da equipe cirúrgica, como da capacitação, competência e atualização dos enfermeiros (GEBRIM, et al., 2016).

O enfermeiro enquanto integrante da equipe de saúde ou em articulação com outros profisisonais podem e devem realizar atividades educativas próprias, a fim de prevenir a ISC. Outras intervenções de enfermagem podem ser implementadas a fim de melhorar a assistência e diminuir os casos de ISC. A confirmação do banho pré-operatório; a verificação da roupa privativa, preparo da equipe cirúrgica em relação às unhas, paramentação, instrumentação; padrões de circulação; controle glicêmico em pacientes diabéticos, controle de fatores ambientais em sala cirúrgica; cuidados com pacientes na sala de recuperação pós-anestésica (SRPA); avaliação do paciente pré alta hospitalar; informações de educação e saúde para pacientes e acompanhantes no pós-operatório domiciliar; cultura para exame microbiológico, se necessário; uso de (EPI's); construção de procedimento operacional padrão (POP) e cheklist, são intervenções a que ajudam a prevenir a ISC e a melhorar sistematicamente a segurança do paciente (ROSCANI, et al., 2015; CARVALHO,et.al., 2015).

Isto posto, verifica-se que apesar de haver um número significativo de estudos abordando a temática, a Sistematização da Assistência de Enfermagem (SAE) deve ser priorizada como um recurso voltado a potencializar a prática do enfermeiro, tornando-o mais qualificado para atender 
as necessidades do indivíduo, além de proporcionar segurança ao paciente submetido a procedimentos cirúrgicos.

\section{Considerações finais}

Identifica-se que a ISC é uma complicação cirúrgica considerada um problema de saúde grave, sendo de grande prevalência hospitalar e tem como causa principal as IRAS. Além de fatores intrínsecos do paciente, esta é causada principalmente por fatores ambientais, envolvendo o local do procedimento cirúrgico, os materiais utilizados, a equipe de saúde, a assistência pós-cirúrgica desde a sala de recuperação pós-anestésica até o período pós-operatório mediato, além da assistência domiciliar.

A ISC pode impactar de modo geral, incluindo o aumento nos gastos de saúde pública devido o maior tempo de internação, além dos danos causados aos pacientes e familiares. Nesse contexto, ressalta-se que o enfermeiro e a equipe assistencial assumem um papel fundamental no processo de cuidado frente a redução na incidência de ISC. Assim, enfatiza-se a atuação do enfermeiro na perspectiva de padronizar produtos e processos para a saúde, na educação continuada, na construção de cheklists, protocolos, guias e indicadores que melhorem a assistência, previnam riscos e garantam a qualidade de vida dos pacientes.

Isto posto, verifica-se que estudos enfocando os cuidados e intervenções específicas de enfermagem frente à prevenção da ISC são relativamente inconclusivos, havendo a necessidade de um maior aprofundamento e sensibilização para o problema, além da otimização de investimentos em pesquisas e ações de educação para capacitar e atualizar cientificamente as equipes, sobretudo 0 enfermeiro enquanto agente transformador de sua prática profissional.

\section{Referências}

ABBAS, Pittet Surgical site infection prevention: a global priority. Journal of Hospital Infection, $\quad$ v.93, $\quad$ p.319-22, $2016 . \quad$ Disponível em:< https://www.journalofhospitalinfection.com/article/S0195-6701(16)30127-X/abstract>. Acesso em: 03 Jan. 2019. 
BELLUSSE, Gislaine Cristhina et al. Fatores de risco de infecção da ferida operatória em neurocirurgia. Acta Paul Enferm, v.28, n.1, p.66-73, 2015. Disponível em:< http://www.scielo.br/pdf/ape/v28n1/1982-0194-ape-028-001-0066.pdf>. Acesso em: 03 Jan. 2019.

BRASIL. Diretrizes Globais para a Prevenção de Infecções de Sítio Cirúrgico. $\begin{array}{llll}\text { Proqualis, } & 2017 . & \text { Disponível } & \text { em;< }\end{array}$ https://proqualis.net/sites/proqualis.net/files/Diretrizes\%20globais\%20para\%20a\%20pr even\%C3\%A7\%C3\%A30\%20de\%20infec\%C3\%A7\%C3\%B5es\%20de\%20s\%C3\%ADt io\%20cir\%C3\%BArgico.pdf>. Acesso em: 03 Jan. 2019.

CARVALHO, Isis Cristiane Bezerra de Melo; SOUZA, Nilba Lima; MEDEIROS, Angélica Tereza Nascimento. Fatores predisponentes para infecção da ferida operatória pós-cesárea: uma revisão integrativa. Fundam. care. Online, v.6, n.2, p.812820, 2014. Disponível em:< https://www.redalyc.org/pdf/5057/505750622037.pdf>. Acesso em: 03 Jan. 2019.

CARVALHO, Vanessa Moura et al. Conhecimento dos profissionais de enfermagem sobre fatores de risco relacionados à infecção de sítio cirúrgico. R. Interd. v. 8, n. 3, p. $1-11$, 2015.

Disponível em:https://revistainterdisciplinar.uninovafapi.edu.br/index.php/revinter/article/view/596.

CARVALHO, Rodrigues et al. Incidência e fatores de risco para infecção de sítio cirúrgico em cirurgias gerais. Rev. Latino-Am. Enfermagem, v.25, p.2848, 2017. Disponível em:< http://www.scielo.br/scielo.php?script=sci_arttext\&pid=S010411692017000100390\&lng=p\&nrm=iso\&tlng=pt>. Acesso em: 03 Jan. 2019.

FRANCO, Lucia Maciel de Castro et al. Efeitos do banho pré-operatório na prevenção de infecção cirúrgica: Estudo clínico piloto. Rev Min Enferm, v.21, p.1053,2017. Disponível em:< http://www.reme.org.br/artigo/detalhes/1191>. Acesso em: 03Jan. 2019.

GEBRIM, Cyanea ferreira lima et al. Indicadores de processo para prevenção da infecção do sítio cirúrgico sob a ótica da segurança do paciente. Enfermeria Global, n.44, $2016 . \quad$ Disponível em:< http://scielo.isciii.es/pdf/eg/v15n44/pt_administracion2.pdf>. Acesso em 03 Jan. 2019. 
MENDES, Karina Dal; SILVEIRA, Renata Cristina Campos Pereira; GALVÃO, Cristina Maria. Revisão integrativa: método de pesquisa para a incorporação de evidências na saúde e na enfermagem. Texto Contexto Enferm,, v.17, n.4, p.758-64, 2008. Disponível em:< http://www.scielo.br/pdf/tce/v17n4/18.pdf>. Acesso em: 03 Jan. 2019.

MARTINS, Tatiana et al. Pré-operatório de cirurgias potencialmente contaminadas: fatores de risco para infecção do sítio cirúrgico. Acta Paul Enferm, v.30, n.1, p.16-24, 2017. Disponível em:http://www.scielo.br/pdf/ape/v30n1/1982-0194-ape-30-010016.pdf. Acesso em: 03 Jan. 2019.

PEREIRA, Hoberdan Oliveira; REZENDE, Maria Edna; COUTO, Bráulio Roberto Gonçalves Marinho. Tempo de internação pré-operatório: um fator de risco para reduzir a infecção cirúrgica em fraturas de fêmur. Revbrasortop, v.50, n.6, p.638-46, 2015. Disponível em:< http://www.scielo.br/pdf/rbort/v50n6/pt_1982-4378-rbort-50-0600638.pdf>. Acesso em: 03 Jan. 2019.

ROSCANI, Alessandra Nazareth Cainé Pereira et al. Validação de checklist cirúrgico para prevenção de infecção de sítio cirúrgico. Acta Paul Enferm, v.28, n.6, p.553-65, 2015. Disponível em:< http://www.scielo.br/pdf/ape/v28n6/1982-0194-ape-28-060553.pdf>. Acesso em: 03 Jan. 2019.

RODRIGUES, André Luiz et al. Avaliação de pacientes quanto à infecção de sítio cirúrgico, em um hospital público de Belém-Pa. Revista paraense de medicina, v.28, n.1, 2014. Disponível em:< http://files.bvs.br/upload/S/01015907/2014/v28n1/a4160.pdf>. Acesso em: Jan. 2019.

ROCHA, Junia Pisaneschi Jardim; LAGES, Clarice Aparecida Simão. O Enfermeiro e a prevenção das infecções do sítio cirúrgico. Cadernos UniFOA, n. 30, p. 117-28, 2016. Disponível em:< https://www.google.com/search?q=O+Enfermeiro+e+a+preven\%C3\%A7\%C3\%A3o+da s+infec\%C3\%A7\%C3\%B5es+do+s\%C3\%ADtio+cir\%C3\%BArgico\&oq=O+Enfermeiro+ e+a+preven\%C3\%A7\%C3\%A3o+das+infec\%C3\%A7\%C3\%B5es+do+s\%C3\%ADtio+ci r\%C3\%BArgico\&aqs=chrome..69i57j69i64.435j0j7\&sourceid=chrome\&ie=UTF-8>. Acesso em: 03 Jan. 2019.

REIS, Raíssa Gabriela; RODRIGUES Maria Cristina Soares Rodrigues. Infecção de sítio cirúrgico pós-alta: ocorrência e caracterização de egressos de cirurgia geral. 
Cogitare enferm. v.22, n.4, p.516-78, 2017. Disponível em:< http://docs.bvsalud.org/biblioref/2017/12/876610/51678-220463-1-pb.pdf>. Acesso em: 03 Jan. 2019.

SANTOS, Gabriela do Carmo et al. Incidência e fatores de risco de infecção de sítio cirúrgico: revisão integrativa. Revista eletrônica do curso de pedagogia/ itinerarius $\begin{array}{lllll}\text { reflectionis, } & \text { v.11, } & \text { n.1, } & \text { Disponível } & \text { em:< }\end{array}$ https://www.revistas.ufg.br/rir/article/view/34142/20357>.

SOARES, Cassia Baldini et al. Revisão integrativa: conceitos e métodos utilizados na enfermagem. Rev Esc Enferm, v.48, n.2, p.335-45, 2014. Disponível em:< http://www.periodicos.usp.br/reeusp/article/view/84097/86950>. Acesso em: 03 Jan. 2019.

SEGRETI, John et al. Introduction to the Centers for Disease Control and Prevention and Healthcare Infection Control Practices Advisory Committee Guideline for Prevention of Surgical Site Infection: Prosthetic Joint Arthroplasty Section. SURGICAL INFECTIONS, $\quad$ v.18, $\quad \mathrm{n} 4, \quad 2017 . \quad$ Disponível em:< https://www.liebertpub.com/doi/pdf/10.1089/sur.2017.068>. Acesso em: 03 Jan. 2019.

TORRES, Lilian Machado et al. Readmissão por infecção do sítio cirúrgico ortopédico: uma revisão integrativa. Rev Esc Enferm USP, v.49, n.6, p.1008-015, 2015. Disponível em:< http://www.scielo.br/pdf/reeusp/v49n6/pt_0080-6234-reeusp-49-06-1008.pdf>. Acesso em: 03 de Jan. 2019. 